\title{
ON THE ABSOLUTE SUMMABILITY BY BOREL'S INTEGRAL METHOD OF THE DERIVED FOURIER SERIES AND ITS CONJUGATE SERIES
}

\author{
by R. M. SHARMA
}

(Received 14th September 1966)

Summary. Mohanty (1) and (3) considered the absolute summability of conjugate series and Fourier series by Borel's integral method by proving the following theorems.

Theorem A. If $\psi(t) \log \frac{k}{t}$ is of bounded variation in $(0, \pi)$, then $\sum_{n=1}^{\infty} B_{n}(\theta)$ is summable $\left|B^{\prime}\right|$.

Theorem B. If $g(t)$ is of bounded variation in $(0, \pi)$, then the series $\sum_{n=1}^{\infty} A_{n}(\theta)$ is summable $\left|B^{\prime}\right|$.

The present author considered the absolute summability of derived Fourier series and its conjugate series by Borel's integral method. Theorems proved by the present author are

Theorem 1. If

(i) $\psi(+0)=0$

and

(ii) $\int_{0}^{\delta} t^{-2}|d \psi(t)|<\infty ; 0<\delta<1$.

then the series $\sum_{1}^{\infty} n B_{n}(\theta)$ is summable $\left|B^{\prime}\right|$.

Theorem 2. If

(i) $\phi(+0)=O(1)$

and

(ii) $\int_{0}^{\delta} t^{-2}|d \phi(t)|<\infty ; 0<\delta<1$.

then the series $\sum_{1}^{\infty} n A_{n}(\theta)$ is summable $\left|B^{\prime}\right|$. 


\section{Definition}

A series $\sum_{0}^{\infty} a_{n}$ is said to be summable $\left(B^{\prime}\right)$ to $\operatorname{sum} A$ if

$$
\int_{0}^{\infty} e^{-x} \cdot \sum_{0}^{\infty} \frac{a_{n} x^{n}}{n !} d x=\lim _{x \rightarrow \infty} \int_{0}^{x} e^{-x} \cdot \sum_{0}^{\infty} \frac{a_{n} x^{n}}{n !} d x=A .
$$

If the above integral is absolutely convergent, we say that the series $\sum_{0}^{\infty} a_{n}$ is absolutely summable by Borel's integral method (2) or summable $\left|B^{\prime}\right|$.

2. Let $f(t)$ be Lebesgue integrable in $(-\pi, \pi)$ and periodic with period $2 \pi$ and let

$$
f(t) \sim \frac{1}{2} a_{0}+\sum_{1}^{\infty}\left(a_{n} \cos n t+b_{n} \sin n t\right)=\frac{1}{2} a_{0}+\sum_{1}^{\infty} A_{n}(t) .
$$

The allied series of (2.1) at $t=\theta$ is

and the derived Fourier series is

$$
\sum_{1}^{\infty}\left(b_{n} \cos n \theta-a_{n} \sin n \theta\right)=\sum_{1}^{\infty} B_{n}(\theta)
$$

$$
\begin{aligned}
& \text { The conjugate series of (2.2) is } \\
& \sum_{1}^{\infty} n B_{n}(\theta) . \\
& \sum_{1}^{\infty} n A_{n}(\theta) . \\
& \phi(t)=\frac{1}{2}\{f(\theta+t)+f(\theta-t)\} \\
& \psi(t)=\frac{1}{2}\{f(\theta+t)-f(\theta-t)\}
\end{aligned}
$$

and

Mohanty (1) and (3) proved

$$
g(t)=\phi(t) \log \frac{k}{t}
$$

Theorem A. If $\psi(t) \log \frac{k}{t}$ is of bounded variation in $(0, \pi)$, then $\sum_{n=1}^{\infty} B_{n}(\theta)$ is summable $\left|B^{\prime}\right|$.

Theorem B. If $g(t)$ is of bounded variation in $(0, \pi)$ then the series $\sum_{n=1}^{\infty} A_{n}(\theta)$ is summable $\left|B^{\prime}\right|$.

3. The object of the present paper is to prove the following two theorems.

Theorem 1. If (i) $\psi(+0)=0$

and (ii) $\int_{0}^{\delta} t^{-2}|d \psi(t)|<\infty ; 0<\delta<1$,

then the series $\sum_{n=1}^{\infty} n B_{n}(\theta)$ is summable $\left|B^{\prime}\right|$. 
Theorem 2. If (i) $\phi(+0)=O(1)$

and (ii) $\int_{0}^{\delta} t^{-2}|d \phi(t)|<\infty ; 0<\delta<1$

then the series $\sum_{n=1}^{\infty} n A_{n}(\theta)$ is summable $\left|B^{\prime}\right|$.

4. In order to simplify the proof we require the following estimates for the function

$$
\begin{aligned}
g_{1}(x, t) & =\int_{t}^{\delta} e^{x \cos u} \cdot \sin (u+x \sin u) d u ; 0<t<\delta<1, x>0 . \\
& =O\left(e^{x}\right) \\
& =O\left(x^{-1} e^{x \cos t}\right) \\
& =O\left(x^{-1} e^{x}\right) \\
g_{2}(x, t) & =\int_{t}^{\delta} e^{x \cos u} \cdot \cos (u+x \sin u) d u ; 0<t<\delta<1, x>0 . \\
& =O\left(e^{x}\right) \\
& =O\left(x^{-1} e^{x \cos t}\right) \\
& =O\left(x^{-1} e^{x}\right)
\end{aligned}
$$

Proof. Let $0<t<\delta<1, x>0$ and $\varepsilon_{1}, \varepsilon_{2}$ be either 0 or 1. Define

$$
h(x, t)=\int_{t}^{\delta} e^{x \cos u} \cdot \sin \left(u+\frac{1}{2} \pi \varepsilon_{1}\right) \sin \left(x \sin u+\frac{1}{2} \pi \varepsilon_{2}\right) d u_{\lambda}
$$

using the second mean value theorem for integrals twice

$$
\begin{aligned}
h(x, t) & =e^{x \cos t} \int_{t}^{s} \sin \left(u+\frac{1}{2} \pi \varepsilon_{1}\right) \sin \left(x \sin u+\frac{1}{2} \pi \varepsilon_{2}\right) d u ;(t<s<\delta) \\
& =x^{-1} \cdot e^{x \cos t} \int_{t}^{s} x \cos u \sin \left(x \sin u+\frac{1}{2} \pi \varepsilon_{2}\right) \sec u \sin \left(u+\frac{1}{2} \pi \varepsilon_{1}\right) d u \\
& =x^{-1} \cdot e^{x \cos t}\left[\varepsilon_{1}+\left(1-\varepsilon_{1}\right) \tan s\right] \int_{r}^{s} x \cos u \sin \left(x \sin u+\frac{1}{2} \pi \varepsilon_{2}\right) d u \\
& =0\left(x^{-1} e^{x \cos t}\right)
\end{aligned}
$$

from which properties (4.2), (4.3), (4.5) and (4.6) follow at once.

\section{Proof of Theorem 1}

E.M.S.-T

$$
\begin{aligned}
& \sum_{n=1}^{\infty} n B_{n}(\theta) \text { is summable }\left|B^{\prime}\right| \text { if } \\
& I=\int_{0}^{\infty} e^{-x}\left|\sum_{1}^{\infty} \frac{n B_{n}(\theta)}{n !} x^{n}\right| d x<\infty .
\end{aligned}
$$


Now

$$
\begin{aligned}
I & =2 \pi^{-1} \int_{0}^{\infty} e^{-x}\left|\sum_{1}^{\infty} \frac{\int_{0}^{\pi} \psi(t) \sin n t}{(n-1) !} x^{n} d t\right| d x \\
& =2 \pi^{-1} \int_{0}^{\infty} e^{-x}\left|\int_{0}^{\pi} \psi(t) \cdot \sum_{1}^{\infty} \frac{x^{n} \sin n t}{(n-1) !} d t\right| d x \\
& =2 \pi^{-1} \int_{0}^{\infty} e^{-x}\left|\int_{0}^{\pi} \psi(t) \cdot x e^{x \cos t} \cdot \sin (t+x \sin t) d t\right| d x \\
& \leqq 2 \pi^{-1} \int_{0}^{\infty} x e^{-x}\left|\int_{0}^{\delta} \psi(t) e^{x \cos t} \cdot \sin (t+x \sin t) d t\right| d x \\
& +2 \pi^{-1} \int_{0}^{\infty} x e^{-x}\left|\int_{\delta}^{\pi} \psi(t) \cdot e^{x \cos t} \cdot \sin (t+x \sin t) d t\right| d x ;(0<\delta<1) \\
& \leqq I_{1}+I_{2}, \text { say }
\end{aligned}
$$

We have

$$
\begin{aligned}
I_{2} & \leqq 2 \pi^{-1} \int_{0}^{\infty} x e^{-x} d x \int_{\delta}^{\pi}|\psi(t)| \cdot e^{x \cos t} d t \\
& \leqq 2 \pi^{-1} \int_{0}^{\infty} x e^{-x} \cdot e^{x \cos \delta} d x \int_{\delta}^{\zeta}|\psi(t)| d t, \quad(\delta<\zeta<\pi) \\
& \leqq 2 \pi^{-1}\left\{\left[\frac{-x e^{-2 x \sin ^{2}} \frac{1}{2} \delta}{2 \sin ^{2} \frac{1}{2} \delta}\right]_{0}^{\infty}+\int_{0}^{\infty} \frac{e^{-2 x \sin ^{2} \frac{1}{2} \delta}}{2 \sin ^{2} \frac{1}{2} \delta} d x\right\} \int_{\delta}^{\zeta}|\psi(t)| d t \\
& \leqq \frac{1}{2} \pi^{-1} \operatorname{cosec}^{4} \frac{1}{2} \delta \cdot \int_{0}^{\pi}|\psi(t)| d t<\infty .
\end{aligned}
$$

Now

$$
\begin{aligned}
& \int_{0}^{\delta} \psi(t) e^{x \cos t} \cdot \sin (t+x \sin t) d t \\
& =-\left[\psi(t) \int_{t}^{\delta} e^{x \cos u} \cdot \sin (u+x \sin u) d u\right]_{0}^{\delta}+\int_{0}^{\delta} d \psi(t) \int_{t}^{\delta} e^{x \cos u} \cdot \sin (u+x \sin u) d u \\
& =\int_{0}^{\delta} d \psi(t) g_{1}(x, t)
\end{aligned}
$$

by condition (i) of the theorem. Therefore

$$
\begin{aligned}
I_{1} & =2 \pi^{-1} \int_{0}^{\infty} x e^{-x}\left|\int_{0}^{\delta} d \psi(t) g_{1}(x, t)\right| d x \\
& \leqq 2 \pi^{-1} \int_{0}^{\delta}|d \psi(t)| \int_{0}^{\infty} x e^{-x}\left|g_{1}(x, t)\right| d x \\
& \leqq 2 \pi^{-1} \int_{0}^{\delta}|d \psi(t)| . J, \text { say }
\end{aligned}
$$


Now

$$
\begin{aligned}
J & =\int_{0}^{1} x e^{-x}\left|g_{1}(x, t)\right| d x+\int_{1}^{t^{-1}} x e^{-x}\left|g_{1}(x, t)\right| d x+\int_{t-1}^{\infty} x e^{-x}\left|g_{1}(x, t)\right| d x \\
& =J_{1}+J_{2}+J_{3}, \text { say }
\end{aligned}
$$

By (4.1) we have

By (4.3) we have

$$
\begin{aligned}
J_{1} & =\int_{0}^{1} x e^{-x} \cdot O\left(e^{x}\right) d x \\
& =O(1)
\end{aligned}
$$

By (4.2) we have

$$
\begin{aligned}
J_{2} & =\int_{1}^{t-1} x e^{-x} \cdot O\left(x^{-1} e^{x}\right) d x \\
& =O\left(t^{-1}\right) .
\end{aligned}
$$

$$
\begin{aligned}
J_{3} & =\int_{t^{-1}}^{\infty} x e^{-x} \cdot O\left(x^{-1} e^{x \cos t}\right) d x \\
& =O\left(\int_{t^{-1}}^{\infty} e^{-2 x \sin ^{2} \frac{1}{2} t} \cdot d x\right) \\
& =O\left(t^{-2}\right)
\end{aligned}
$$

There is therefore an $A$ with

Thus the theorem is proved

$$
I \leqq I_{1}+I_{2} \leqq A+A \int_{0}^{\delta} t^{-2}|d \psi(t)|<\infty .
$$

\section{Proof of Theorem 2}

Now

$$
\begin{gathered}
\sum_{n=1}^{\infty} n A_{n}(\theta) \text { is summable }\left|B^{\prime}\right| \text { if } \\
I^{\prime}=\int_{0}^{\infty} e^{-x}\left|\sum_{1}^{\infty} \frac{n A_{n}(\theta)}{n !} x^{n}\right| d x<\infty .
\end{gathered}
$$

$$
\begin{aligned}
I^{\prime} & =2 \pi^{-1} \int_{0}^{\infty} e^{-x}\left|\sum_{1}^{\infty} \frac{\int_{0}^{\pi} \phi(t) \cos n t d t}{(n-1) !} x^{n}\right| d x \\
& \leqq 2 \pi^{-1} \int_{0}^{\infty} x e^{-x}\left|\int_{0}^{\delta} \phi(t) e^{x \cos t} \cdot \cos (t+x \sin t) d t\right| d x \\
& +2 \pi^{-1} \int_{0}^{\infty} x e^{-x}\left|\int_{\delta}^{\pi} \phi(t) e^{x \cos t} \cdot \cos (t+x \sin t) d t\right| d x \\
& \leqq I_{1}^{\prime}+I_{2}^{\prime}
\end{aligned}
$$


say, where $0<\delta<1$ and

$$
I_{2}^{\prime} \leqq 2 \pi^{-1} \int_{0}^{\infty} x e^{-x} \cdot e^{x \cos \delta} d x \int_{\delta}^{\xi}|\phi(t)| d t ;(\delta<\zeta<\pi)<\infty
$$

Now

$$
\int_{0}^{\delta} \phi(t) e^{x \cos t} \cdot \cos (t+x \sin t) d t=O(1)+\int_{0}^{\delta} d \phi(t) g_{2}(x, t)
$$

by condition (i) of the theorem. Therefore

$$
\begin{aligned}
I_{1}^{\prime} & \leqq 2 \pi^{-1} \int_{0}^{\delta}|d \phi(t)| \cdot \int_{0}^{\infty} x e^{-x} \cdot\left|g_{2}(x, t)\right| d x+A \\
& \leqq 2 \pi^{-1} \int_{0}^{\delta}|d \phi(t)| \cdot J^{\prime}+A, \text { say }
\end{aligned}
$$

Proceeding as in the proof of Theorem 1 and using (4.4), (4.5) and (4.6) we have

$$
J^{\prime}=O\left(\boldsymbol{t}^{-2}\right)
$$

Therefore

$$
I^{\prime} \leqq A+A \int_{0}^{\delta} t^{-2}|d \phi(t)|<\infty
$$

Thus the theorem is proved.

I am much indebted to Dr. B. D. Singh, Head of the Mathematics Department of the University of Saugar and to the referee for valuable suggestions.

\section{REFERENCES}

(1) R. MOHANTY, The absolute summability by Borel's integral method of the allied series of a Fourier series, J. London Math. Soc. 38 (1963), 381-384.

(2) G. H. HaRdy, Divergent Series (Oxford, 1949), p. 184.

(3) R. MOHANTY, On the summation of Fourier series by Borel's absolute integral method, J. London Math. Soc. 38 (1963), 375-380.

GovernMENT COLLEGE

SATNA (M.P.)

INDIA 